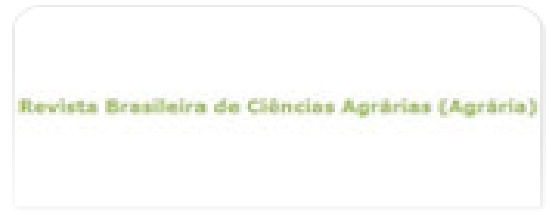

Revista Brasileira de Ciências Agrárias

ISSN: 1981-1160

editorgeral@agraria.pro.br

Universidade Federal Rural de Pernambuco

Brasil

Canul Solis, Jorge R.; Pelcastre Ortega, Arturo; Duarte Vera, Fernando J.

Efecto de Zeranol en solución oleosa sobre el comportamiento de corderos Pelibuey en engorda

Revista Brasileira de Ciências Agrárias, vol. 4, núm. 4, octubre-diciembre, 2009, pp. 485-488

Universidade Federal Rural de Pernambuco

Pernambuco, Brasil

Disponible en: http://www.redalyc.org/articulo.oa?id=119012569019

Cómo citar el artículo

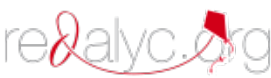

- Número completo

- Más información del artículo

Página de la revista en redalyc.org

Sistema de Información Científica

Red de Revistas Científicas de América Latina, el Caribe, España y Portugal

Proyecto académico sin fines de lucro, desarrollado bajo la iniciativa de acceso abierto 
AGRÁría.

Revista Brasileira de Ciências Agrárias

v.4, n.4, p.485-488, out.-dez., 2009

Recife, PE, UFRPE. Www.agraria.ufrpe.br

Protocolo 597 - 22/05/2009 - Aprovado em 20/07/2009

Jorge R. Canul Solis ${ }^{1}$

Arturo Pelcastre Ortega ${ }^{1}$

Fernando J. Duarte Vera ${ }^{1}$

\section{Efecto de Zeranol en solución oleosa sobre el comportamiento de corderos Pelibuey en engorda}

\section{RESU MEN}

Tradicionalmente, la aplicación de Zeranol en rumiantes se lleva a cabo con implantes en pellets; sin embargo la presentación en implante líquido facilitaría su aplicación. El trabajo se realizó de febrero a mayo de 2004 en Yucatán, M éxico. Se evaluó el efecto del Zeranol en solución oleosa (Z-oleo), aplicado por vía subcutánea, sobre la ganancia diaria de peso (GDP), consumo voluntario (CV) y conversión alimenticia (CA) de ovinos Pelibuey en engorda intensiva, durante 56 días. Se utilizaron 32 machos enteros, con peso promedio de $19.8 \pm 2.7 \mathrm{~kg}$ y 146 días de edad, aleatorizados en dos tratamientos $(\mathrm{T} 1=\sin \mathrm{Z}$-oleo y $\mathrm{T} 2=$ con Z-oleo). El día cero los animales del $\mathrm{T} 2$ recibieron dosis única por vía subcutánea de $12 \mathrm{mg}$ de Z-oleo. Los ovinos se alojaron en corraletas individuales, recibiendo una dieta integral a libre acceso. No se observaron diferencias estadísticas entre tratamientos en las variables analizadas ( $P>0.05$ ). Se obtuvieron GDP de $323 \pm 51$ y $331 \pm 38 \mathrm{~g} \mathrm{día}^{-1}$; CV de $1.575 \pm 0.203$ y $1.644 \pm 0.193 \mathrm{Kg}$ de MS d-1 y CA mostró medias de $4.9 \pm 0.4$ y $5.0 \pm$ 0.6 para T1 y T2, respectivamente. El uso del Zeranol en solución oleosa, no tuvo efecto sobre ganancia diaria de peso, consumo voluntario y conversión alimenticia en ovinos Pelibuey en engorda intensiva.

Palabras clave: 0 vinos de pelo, implantes, ganancia de peso, consumo voluntario, conversión alimenticia

\section{Effect of Zeranol in oil solution on feedlot performance of Pelibuey lambs}

\begin{abstract}
Traditionally, the application of Zeranol in ruminants is carries out with implants in pellets; nevertheless its presentation in oily suspension would facilitate its application. The work was carried out from February to May of 2004 in Yucatan, M exico. The effect of the Zeranol in oily solution (Z-olea) was evaluated on the daily weight gain, voluntary feed intake, and feed efficiency in Pelibuey feedlot lambs. Thirty two Pelibuey lambs were used, with a weight average of $19.8 \pm 2.7 \mathrm{~kg}$ and 146 days of age, allotted randomly to the two treatments ( $T 1=$ no application of $Z$ and $T 2=$ with application), the lambs received a unique subcutaneous dose of $12 \mathrm{mg}$ of Z-olea; each lamb was considered an independent experimental unit. The lambs were lodged separately, with free feed access for 56 days. Statistical differences were not observed between treatments in any variables ( $>>0.05)$. The lambs had a daily weight gain of $323 \pm 51$ and $331 \pm 38 \mathrm{~g} \mathrm{day}^{-1}$; voluntary feed intake of $1.575 \pm 0.203$ and $1.644 \pm 0.193 \mathrm{~kg}$ dry matter day ${ }^{-1}$ and feed efficiency of $4.9 \pm 0.4$ and $5.0 \pm 0.6$ for T1 and T2, respectively. Under the conditions in which this work was performed, the results indicated that Zeranol injected in oily solution, did not affect the daily weight gain, voluntary feed intake and feed efficiency in Pelibuey feedlot lambs.
\end{abstract}

Key words: Hair lambs, implants, daily weight gain, intake voluntary, feed efficiency 


\section{INTRODUCCIÓN}

Los aditivos en la alimentación animal, en particular los anabólicos, incrementan la tasa de crecimiento, favorecen la producción de músculo magro y mejoran la conversión alimenticia (CA), cuando son utilizados de manera apropiada (Morón \& Rumbos, 1997). Sin embargo, la respuesta a la aplicación de los aditivos depende de la calidad genética del animal, la alimentación, estado de salud, entre otras.

En novillas implantadas con Zeranol, se ha encontrado mejora en la tasa de crecimiento, comparado con animales no implantados (Nichols \& Lesperance, 1973).

La aplicación de Zeranol en la fase de finalización de ovinos permite incrementar la ganancia diaria de peso (GDP) en las razas lanares (Gómez et al., 2004); de igual forma, en ovinos cruzados se incrementa la GDP (20\%) y la CA (17\%), cuando son implantados repetidamente con Zeranol (Hufstedler et al., 1996). Similarmente con ovinos cruzados, donde se estudió la interacción de los niveles de $\mathrm{P}$ y Ca en la dieta, se mejoró la GDP en $26 \%$ y en $12 \%$ la CA en los animales implantados con Z, en comparación con los no implantados (Hutcheson et al., 1992).

En el trópico, con ovinos de pelo en sistemas bajo pastoreo, se obtuvieron GDP entre 29 y $78 \mathrm{~g} \mathrm{~d}^{-1}$ (Hernández et al., 2000). En sistemas con alimentación intensiva a base de dietas integrales, se pueden obtener GDP posdestete entre 108 y 276 g (Duarte \& Pelcastre, 1998).

Generalmente, el Zeranol es aplicado a través implantes subcutáneos mediante pellets. Sin embargo, la presentación de Zeranol en solución oleosa (Zeramec ®), ha sido utilizada con bovino obtiendo resultados favorables, mejorando la ganancia de peso, el consumo voluntario y la conversión alimenticia en la engorda de becerros (Palomino et al., 2004).

Se han realizado trabajos sobre el uso de implantes en pellets en la engorda de bovinos y ovinos; sin embargo, el uso y efecto de este Zeranol en solución oleosa inyectable no ha sido estudiado en ovejas de pelo tropical, con base a lo anterior, el objetivo del presente estudio fue determinar el efecto del Zeranol en solución oleosa inyectable sobre la ganancia diaria de peso, el consumo voluntario y la conversión alimenticia de ovinos Pelibuey en engorda intensiva.

\section{MATERIAL Y METODOS}

El trabajo se desarrolló en el Campo Experimental Tizimín, perteneciente al Instituto Nacional de Investigaciones Forestales, Agrícolas y Pecuarias (INIFAP), en la zona oriente de Yucatán; México., a $21^{\circ} 10^{\prime}$ latitud norte y $87^{\circ} 57^{\prime}$ longitud oeste. De acuerdo a la clasificación de Köppen, la zona cuenta con un clima Aw0 (cálido subhúmedo con lluvias en verano); el promedio de la precipitación pluvial anual es de 1056 $\mathrm{mm}$, distribuida en los meses de junio a noviembre; una temperatura anual media de $24.7^{\circ} \mathrm{C}$ y una altura de $15 \mathrm{msnm}$.

Se utilizaron 32 ovinos machos de la raza Pelibuey, sin castrar, con peso promedio inicial de $19.8 \pm 2.7 \mathrm{~kg}$ y edad promedio de 146 días; los animales se distribuyeron en dos grupos, en un diseño completamente al azar, con 16 repeticiones por tratamiento, considerando cada ovino como una unidad experimental. Los tratamientos fueron: T1 $=$ sin aplicación de Zeranol $(Z)$ y T2 = aplicación de una dosis única de $12 \mathrm{mg}$ de Zeranol solución oleosa (Z-oleo) por vía subcutánea (Zeramec $\left.{ }^{\circledR}\right)$, al inicio del estudio. El anabólico en solución oleosa en el T2, se empleó combinado con Ivermectina, a dosis de $0.2 \mathrm{mg}$ animal ${ }^{-1}$, por lo que en T1 se utilizó un endectocida homólogo (Moxidectina) a la misma dosis, el cual actúa de forma similar sobre el acido gammaaminobutírico (GABA).

Previo a la fase experimental, los animales se sometieron a un periodo de ocho días de adaptación a la dieta integral con $15 \%$ de proteína bruta (PB) y $2.7 \mathrm{Mcal} \mathrm{EM} \mathrm{kg}^{-1}$ (Tabla 1). Posteriormente, recibieron el tratamiento correspondiente, considerándose este momento como el inicio del trabajo experimental.

Tabla 1. Composición de la dieta integral para ovinos Pelibuey tratados con Zeranol en solución oleosa

Table 1. Integral diet composition for Pelibuey lambs implanted with Zeranol in oil solution

\begin{tabular}{lc}
\hline \multicolumn{1}{c}{ Ingredientes } & Porcentaje \\
\hline Pasta de soya & 5 \\
Sorgo común (grano). & 44 \\
Heno de pasto llanero (Andropogon gayanus Kunth) & 9 \\
Melaza de caña de azúcar & 17 \\
Pollinaza & 25 \\
\hline
\end{tabular}

Niveles de garantía $/ \mathrm{kg}: \mathrm{PB}=150 \mathrm{~g} ; \mathrm{EM}=2.7 \mathrm{Mcal} ; \mathrm{Ca}=27.9 \mathrm{~g} ; \mathrm{P}=22.5 \mathrm{~g} ; \mathrm{FC}=53 \mathrm{~g}$;

MS $=78.66 \%$; Contenido estimado de acuerdo a la base de datos de insumos reportados en la literatura (NRC, 1985; Tedeschi et al., 2002)

Los ovinos se alojaron en corraletas individuales de 4.8 $\mathrm{m}^{2}$, con comedero y bebedero, con piso de tierra, realizando el aseo de excretas cada ocho días. El alimento se proporcionó a partir de $1.2 \mathrm{~kg}$ animal ${ }^{-1} \mathrm{dí}^{-1}$, incrementando la cantidad ofrecida conforme aumentaba el consumo, adicionando para el día siguiente un excedente de $200 \mathrm{~g}$ a partir del alimento consumido; de igual forma recibieron sales minerales y agua fresca a libre acceso. El trabajo tuvo una duración de 56 días.

Los animales se pesaron cada catorce días en una báscula de plataforma sin ayuno previo, con la adaptación de una jaula metálica; para calcular la ganancia diaria de peso (GDP), se obtuvo la diferencia entre el peso final y el inicial, dividido entre los días correspondientes al período. Se calculó el consumo voluntario $(\mathrm{CV})$, mediante la diferencia entre el alimento ofrecido y rechazado; la conversión alimenticia (CA) se obtuvo a partir de la cantidad total de alimento consumido en el intervalo entre la ganancia de peso durante el mismo período.

La GDP, CV y CA promedios por tratamiento fueron sometidos a un Análisis de Varianza, utilizando el siguiente modelo estadístico:

$$
Y i j=\grave{i}+T i+A j+a ̊ i j
$$

Donde:

Yij = Respuesta de la aplicación de Z, en el i-ésimo tratamiento, en la j-ésima repetición.

ì = Media teórica poblacional.

$\mathrm{Ti}=$ Efecto del $\mathrm{i}$-ésimo tratamiento. $(\mathrm{i}=1,2)$

$\mathrm{Aj}=$ Efecto de la j-ésima repetición. $(\mathrm{j}=1, \ldots, 16)$

åij = Error experimental, aleatorio e independiente distribuido con media y varianza cero $\left(\hat{o}^{2}=0\right)$. 
La comparación de medias se realizó mediante la T de Student, utilizando el paquete estadístico SAS (Camacho et al., 1992).

\section{RESULTADOS Y DISCUSIÓN}

No se observó efecto del Z-oleo sobre la ganancia diaria de peso $(\mathrm{P}>0.05$. Sin embargo, se presentó un pico de producción a los 28 días, con GDP de 342 y 369 g día $^{-1}$ animal $^{-1}$ para los animales sin y con inyección de Z-oleo, respectivamente (Tabla 2).

Tabla 2. Ganancia diaria de peso (g) de ovinos Pelibuey tratados con Zeranol inyectable en solución oleosa

Table 2. Daily weight gain ( $\mathrm{g}$ ) of Pelibuey lambs implanted with Zeranol in oil solution

\begin{tabular}{ccc}
\hline Tratamiento & $\mathbf{n}$ & GDP $(\mathbf{g}) \pm \mathbf{E E}$ \\
\hline Sin Zeranol & 16 & $323 \pm 12$ NS \\
Con Zeranol & 16 & $334 \pm 9$ NS \\
\hline n: número de repeticiones; EE: Error estándar de la media; NS: No significativo (P>0.05)
\end{tabular}

El comportamiento de los ovinos Pelibuey inyectados con Z-ole, en este trabajo, coincide con lo reportado utilizando Z en pellets a la misma dosis en ovinos bajo pastoreo, no observando efecto del implante sobre la ganancia diaria de peso, obteniendo $145 \mathrm{~g}$ animal $^{-1}$ día $^{-1}$ (Hervé \& Throm, 1984; Olivares, 1990; Oliva \& Vidal, 2001; Dzul, 2004); de igual forma, Gómez et al. (2004) reportan comportamientos similares en ovinos F1 de razas cárnicas (Katahdin x Dorper). Sin embargo, en otros estudios con ovinos cruzados, se han observado incrementos de $20 \%$ en la GDP (Hufstedler et al., 1996) y en razas lanares se incrementa entre 14 y $15 \%$, con la aplicación de Z (Wiggins et al., 1979).

Por otro lado, los índices de producción reportados para ovinos Pelibuey y Blackbelly engordados intensivamente bajo condiciones tropicales del Sureste de México, han sido bajos en comparación con los obtenidos en las razas de lana, principalmente por el factor genético de los animales. Sin embargo, en el presente estudio las GDP fueron de 323 y $334 \mathrm{~g}$ día $^{-}$ ${ }^{1}$ para los ovinos Pelibuey sin y con Z-oleo, respectivamente; los cuales son superiores a las reportadas en un rango de 200 a 240 g día $^{-1}$ (Duarte \& Pelcastre, 1998) y en pastoreo con implante de pellets de $\mathrm{Z}$ en ovinos Pelibuey, con valores de $145 \mathrm{~g} \mathrm{día}^{-1}$ (Oliva \& Vidal, 2001). Esto coincide con la literatura, en la que se menciona que el ritmo de crecimiento y la composición del cuerpo se determinan parcialmente por los factores genéticos y la influencia de las hormonas endógenas (Isaza \& González, 1985) y el estrés medioambiental, cuando existe una correlación negativa entre la elevada humedad relativa $(70-95 \%)$ y la temperatura moderada $\left(25-35^{\circ} \mathrm{C}\right)$, con el consumo de alimento y el comportamiento productivo de los ovinos (Bianca, 1972), obteniendo menores GDP.

El consumo voluntario fue similar entre tratamientos $(\mathrm{P}>$ 0.05). Se observó que el consumo voluntario se incrementó conforme aumentaba la edad de los animales en ambos grupos, iniciando con 1.226 hasta $1.718 \mathrm{~kg}$ de MS día ${ }^{-1}$ y de 1.267 a $1.883 \mathrm{~kg}$ de MS día ${ }^{-1}$ para el grupo sin y con Z-oleo, respectivamente (Tabla 3 ).

Tabla 3. Consumo diario de alimento de ovinos Pelibuey tratados con Zeranol en solución oleosa

Table 3. Daily feed intake of Pelibuey lambs implanted with Zeranol in oil solution

\begin{tabular}{ccc} 
Tratamiento & $\mathbf{n}$ & CV $(\mathbf{k g ~ d i ́ a - 1}) \pm$ EE \\
Sin Zeranol & 16 & $1.574 \pm 0.050^{\mathrm{NS}}$ \\
Con Zeranol & 16 & $1.636 \pm 0.047$ NS \\
\hline
\end{tabular}

n: número de repeticiones; EE: Error estándar de la media; NS: No significativo (P>0.05)

Rincón et al. (1994) reportan CV similares a los observados en el presente estudio, con el uso de dos concentrados energéticos (3.4 y $2.5 \mathrm{Mcal} \mathrm{EM} \mathrm{kg}^{-1}$ ) y el uso de $\mathrm{Z}$ a la misma dosis, en animales de pelo (Celorio, 1982; Nahed et al., 1990; Oliva \& Vidal, 2001). Estos reportes evidencian que el uso de $\mathrm{Z}$ en pellets y en solución oleosa inyectable en ovinos de pelo, no tienen efecto sobre el CV de alimento y quizá el incremento en el consumo se deba principalmente a una sincronía en el contenido de energía y nitrógeno en la dieta del animal (Van Söest, 1994) o a la mayor capacidad ruminal y la edad de los animales (Isaza \& González, 1985). Los resultados al respecto no son consistentes ya que existen reportes donde en ovinos cruzados no se ha observado efecto en el CV (Gómez et al., 2004), existiendo reportes donde se ha mejorado hasta en $12 \%$ con el uso de $\mathrm{Z}$ en pellets (Hutcheson et al., 1992).

El uso de Z-oleo no afectó la CA (P > 0.05). Se observa que los corderos a menor edad presentaron una CA más alta en ambos grupos (Tabla 4).

Tabla 4. Conversión alimenticia en ovino Pelibuey tratados con Zeranol en solución oleosa

Table 4. Feed conversion of Pelibuey lambs implanted with Zeranol in oil solution

\begin{tabular}{ccc}
\hline Tratamiento & $\mathbf{n}$ & CA \pm EE \\
\hline Sin Zeranol & 16 & $4.907 \pm 0.101$ NS \\
Con Zeranol & 16 & $4.930 \pm 0.144$ Ns \\
\hline
\end{tabular}

n: número de repeticiones; EE: Error estándar de la media; NS: No significativo ( $P>0.05)$

En la CA, se coincide con resultados reportados en ovinos Pelibuey en crecimiento, bajo estabulación y recibiendo concentrados entre 15 y $17 \%$ de PC, con CA de 5.45 a 5.90 (Borbolla et al., 2004), siendo los resultados similares a los reportados en corderos $\mathrm{F} 1$ de razas lanares y de pelo, cuando se implantan con $\mathrm{Z}$ en pellets (Gómez et al., 2004). Por otra parte, se observa mayor eficiencia a menor edad (160 días comparado contra 194 días), comportamiento semejantes se han reportado en ovinos Pelibuey en crecimiento de entre 20 y $55 \mathrm{~kg}$, en donde los machos, con un peso vivo de 20 a $30 \mathrm{~kg}$, duplicaron la eficiencia en la utilización de la energía para el incremento de peso vivo, comparados con ovinos de 45 a $55 \mathrm{~kg}$ (Partida \& Martínez, 1992). Sin embargo, en ovinos de razas lanares cruzados e implantados con $\mathrm{Z}$ en pellets se reporta mejora en la eficiencia alimenticia hasta en $13 \%$, comparados a los no implantados (Hutcheson et al., 1992). 
En ovinos, no se cuentan con resultados contundentes sobre indicadores económicos del uso de Zeranol; sin embargo, en bovinos en pastoreo se obtiene incrementos en los ingresos netos de 3.5 \% (Morón-Fuenmayor et al., 1999).

\section{CONCLUSIONES}

El uso de Zeranol en solución oleosa inyectable, a dosis de $12 \mathrm{mg}$ por vía subcutánea, en ovinos Pelibuey no afectó la ganancia diaria de peso, el consumo voluntario y la conversión alimenticia.

\section{LITERATURA CITADA}

Bianca, W. Termorregulación. In: Haféz, E.S.E. (Ed.). Adaptación de los animales de granja. México: Ed. Herrera, 1972. p. 135-162.

Borbolla, I.J.E.; Barajas, C.R.; Gaxiola. C.S.M. Efecto de la metionina de cromo $(0.4 \mathrm{ppm})$ en respuesta productiva y características de la canal de ovinos Pelibuey en engorda. En: Reunión Nacional de Investigación Pecuaria, 40, Mérida, 2004. Memoria... Mérida: Universidad Autónoma de Yucatán, 2004. p.289.

Camacho, C.O.: Valle, D.H. del; Ruelas, A.G.A. Statistical Analysis System para Microcomputadoras. Chapingo: s.n., 1992. 174p.

Celorio, D.F.A. Comportamiento del borrego Tabasco en la fase de finalización con implantes hormonales y anabólicos, versus suplementados y no suplementados. México: Universidad Juárez. Autónoma de Tabasco, 1982. Tesis de licenciatura.

Duarte, V. F.; Pelcastre, A. La yuca (Manihot esculenta) como fuente energética en dietas integrales para engorda de borregos Pelibuey y su cruza con Hampshire. Técnica Pecuaria en México, v.36, n.2, p.173-178, 1998.

Dzul, J.R. Evaluación de dos implantes anabólicos sobre los índices productivos en ovinos en el estado de Yucatán. Tizimín: Instituto Tecnológico Agropecuario, 2004. Tesis de licenciatura.

Gómez, A.M.A.; Dávila, F.J.I.; Escobar, M.F.J. Ganancia de peso de ovinos tratados con Zeranol. Veterinaria Zacatecas, v.3, n.2, p.167-172, 2004.

Hernández, M.O.; Pérez, P.J.; Martínez, H.P.A.; Herrera, H.J.G.; Mendoza, M.G.D.; Hernández, G.A. Pastoreo de Kikuyo (Pennisetum clandestinum Hochts.) por borregos en crecimiento a diferentes asignaciones de forraje. Agrociencia, v.34, n.2, p.127-134, 2000.

Hervé, M.; Throm, E. Efectos del Zeranol y complejo vitamínico ADE sobre el crecimiento de corderos en pastoreo. Archivos de Zootecnia. v.33, n.127, p.257-263, 1984.

Hufstedler, G.D.; Gillman, P.L.; Carstens, G.E.; Greene, L.W.; Turner, N.D. Physiological and hormonal responses of lambs repeatedly implanted with Zeranol and provided two levels of feed intake. Journal of Animal Science, v.74, n.10, p.2376-2384, 1996.
Hutcheson, J.P.; Greene, L.W.; Carstens, G.E.; Byers, F.M. Effects of Zeranol and two dietary levels of calcium and phosphorus on performance, carcass and bone characteristics, and calcium status in growing lambs. Journal of Animal Science, v.70, n.5, p.1346-1351, 1992.

Isaza, G; González, J. Efecto de Zeranol y estradiol 17â sobre el peso al destete en terneros cruzados. Palmira: Universidad Nacional de Colombia, 1985. Tesis de licenciatura.

Morón, F.O.E.; Rumbos, J.L.G. Uso de agentes anabólicos en el crecimiento de toretes mestizos comerciales como estrategia de manejo en sabanas Venezolanas. Archivos Latinoamericanos de Producción Animal, v.5, supl.1, p.183, 1997.

Morón-Fuenmayor, O. E.; Pietrosemoli, S.; Aranguren, J. A.; Fossi, A. Uso de agentes anabolizantes solos o combinados sobre el crecimiento de novillos a pastoreo. Revista Científica FCV-LUZ; v.9, n.4, p.299-304, 1999.

Nahed, J.S.; González, R.; Herrera, R.; Barcenas, G.; Mendoza, C.; García, C. Effect of supplementation strategy and Zeranol implant on growing lambs. Journal de Animal Science, v. 68, n.1, p. 528-529, 1990.

Nichols, N.E.; Lesperance, A.L. Type, level and frequency of implant on suckling calves. Journal of Animal Science; v.36, p.1206, 1973.

National Research Council - NRC. Daily nutrient requeriments of sheep. $6^{\text {th }}$ ed.. Washington: NRC, 1985. p. 45-46.

Oliva, H.J.; Vidal, B.A. Utilización de Zeranol en borregos Pelibuey en pastoreo y con concentrado energético. Universidad y Ciencia, v.17, n.34, p.57-64, 2001.

Olivares, V.H., Hallford, D.M. Growth and carcass characteristics and serum growth hormone, prolactin and insulin profiles in Debouillet lambs hormone and Zeranol. Journal of Animal Science, v.68, n.7, p.1971-1979,1990.

Palomino, M.A.I.; Solís, R.; Dávila, I.; Escobar, J. Ganancia de peso de becerros y novillos tratados con Synovex M y Zeramec. Veterinaria Zacatecas, v.3, n.2, p.173-178, 2004.

Partida de la, P.J.A.; Martínez, R.L. Comportamiento de borregos Pelibuey alimentados con dos niveles de energía en cuatro períodos de crecimiento. Técnica Pecuaria en México; v.30, n.1, p.1-11, 1992.

Rincón, C.X.; Moreno, F.; Rosero, O. Efecto de los agentes anabólicos en ovinos tropicales alimentados con dos niveles de energía y proteína. En: Congreso Nacional de Producción Ovina, 7, Toluca, 1994. . Toluca: AMTEO-UNAM, 1994. p. 77-80.

Tedeschi, L.O.; Fox, D.G.; Pell, A.N.; Duarte, L.D.P.; Boin, C.; Development and evaluation of a tropical feed library for the Cornell Net Carbohydrate and Protein System Model. Scienta Agricola, v.59, n. 1, p.1-18, 2002.

Van Söest, P.J. Nutritional Ecology of the Ruminant. 2ed. London: Cornell University Press, 1994. p. 77.

Wiggins, J.P.; Rothenbacher, H.; Wilson, L.; Martin, J.; Wangsness, J.; Ziegler, H. Growth and endocrine responses of lambs to Zeranol implants: Effect of preimplant growth rate and breed of sire. Journal of Animal Science, v.49, n.2, p.291-297, 1979. 